\title{
Advanced planning and scheduling system with application in the tobacco industry
}

Haijun Zhang* and Qiong Yan

School of Mechatronics Engineering, Zhengzhou University of Aeronautics, Zhengzhou, Henan 450046, China

Email: tengda83@163.com

Email: joan2055@163.com

*Corresponding author

\section{Xiying Peng}

School of Information \& Business, Zhongyuan University of Technology, Zhengzhou, Henan 450007, China

Email: nodone@163.com

\begin{abstract}
For the reasonable and real-time planning, this paper proposes a case study of an advanced planning and scheduling system called APSoCT, which is developed for the tobacco industry. Firstly, the cut tobacco processing is modelled. Then, the function modules, the planning process and the intelligent heuristic are designed for APSoCT. The optimisation objectives included the changeover, the shift balance, the facility utilisation, the continuous-batch production and so on. Finally, APSoCT is developed to verify the system design is feasible and reliable. The APSoCT system fulfils the requirements of cut tobacco flow shop in the tobacco industry.
\end{abstract}

Keywords: advanced planning and scheduling system; APS; genetic algorithm; cut tobacco; manufacturing service.

Reference to this paper should be made as follows: Zhang, H., Yan, Q. and Peng, X. (2017) 'Advanced planning and scheduling system with application in the tobacco industry', Int. J. Service and Computing Oriented Manufacturing, Vol. 3, No. 1, pp.24-42.

Biographical notes: Haijun Zhang received his $\mathrm{BS}$ and $\mathrm{PhD}$ degrees from the School of Mechanical and Electronic Engineering, Wuhan University of Technology, Wuhan, China in 2005 and 2010, respectively. He is currently a researcher in the School of Mechatronics Engineering, Zhengzhou University of Aeronautics, Zhengzhou, China. His research interests include genetic algorithms, scheduling problems and advanced planning and scheduling.

Qiong Yan received his MS degree from the School of Mechanical and Electronic Engineering, Wuhan University of Technology, Wuhan, China in 2008. She is currently a Lecturer at the School of Management Engineering, Zhengzhou University of Aeronautics, Zhengzhou, China. Her research interests include project management, JIT and APS. 
Xiying Peng received his MS degree from the Zhongyuan University of Technology, Zhengzhou, China in 2009. She is currently a Lecturer at the School of Information \& Business, Zhongyuan University of Technology, Zhengzhou, China. Her research interests include automatic control, genetic algorithms and scheduling problems

\section{Introduction}

Tobacco products have dual attributes including free-market commodity and monopoly. Not only must the tobacco products follow the free market rule, but also the tobacco industry is a monopoly industry exercising exclusive state trading and selling in most countries. The tobacco industry has leaped to one pillar industry in the national economy. Under the influence of factors that tariff concession, quota, licensing system loosening and monopoly sales cancelling gradually, tobacco market open further, the tobacco industry cannot rely on the support and protection of national monopoly system. Tobacco manufacturers have recently faced intense global competition and declining profits.

In the tobacco industry, a major requirement by the management for efficient production is that production planning and scheduling. Many tobacco manufacturers tend to think that enterprise requirements planning (ERP) system will solve their planning issues. Yet, despite its name, ERP systems are usually transaction-based systems rather than planning systems (Chen, 2001). Traditional production planning methods, such as material requirements planning (MRP), consider only the availability of materials and totally ignore factors such as capacity limits and supply chain configurations (Caridi et al., 2000). The advanced planning and scheduling (APS) system deals with effectively allocating manufacturing resources to complete the multi-level products so that multi-constraints are satisfied and production objectives are met (Moon et al., 2004; Lin et al., 2007).

This paper presents a case study for the APS system for cut tobacco (APSoCT) in the tobacco industry. The proposed APSoCT system explicitly considers due dates of cut tobacco, operation sequences among batches and capacity constraints of the manufacturing system. The optimisation objectives included the changeover, the shift balance, the facility utilisation, the continuous-batch production and so on. An intelligent heuristic is applied to the APSoCT system and the results demonstrate that significant production performances can be achieved while ensuring customer satisfaction as opposed to normal practices followed in the company relying on human expertise.

This paper is organised as follows: Section 2 distinguishes this paper from the existing body of related literature. Section 3 describes the tobacco industrial problem in detail. In Section 4, the function modules of the APS system are designed, the procedure of planning and scheduling is implemented for APSoCT in the tobacco industry and the mathematical formulation for the cut tobacco process is modelled. The genetic algorithm (GA) is applied into the APSoCT system in Section 5. Section 6 displays the development of the APSoCT system for cut tobacco in the tobacco industry. Section 7 concludes this paper with some remarks. 


\section{Literature}

Much research about APS is to solve the shop floor scheduling problem. Yun and Gen (2002) considered a pre-emptive and non-pre-emptive scheduling model as one of the advanced scheduling problems considered by a constraint programming technique and propose a new GA considering simultaneously the pre-emptive and non-pre-emptive cases of the activities of jobs under single machine job-shop scheduling problems. Liu et al. (2009) presented a multi-objective GA with local search for the APS problem. Lei and Wang (2011) considered the scheduling problem of flow shop with many batch processing machines, which were an integral part of many industries such as semiconductor burn-in operations, process industries (tobacco industry), wafer fabrication process, testing electrical circuits and metal working and proposed an effective neighbourhood search algorithm for the problem. Dai et al. (2013) proposed an energy-efficient model for production scheduling problem and adopted an improved genetic-simulated annealing (SA) algorithm to implement a feasible scheduling. Cheng et al. (2014) considered the problem of scheduling two batch-processing machines in flow-shop where the jobs have arbitrary sizes and the machines have limited capacity, which is similar to the tobacco industrial problem in this paper.

Little research is about the development of APS with application in the real world. Pongcharoen et al. (2004) developed a GA-based tool which included a repair process to rectify infeasible schedules. Hashemipour (2004) developed a computer-aided process planning tool (PPSGAR), which was implemented successfully in a firm in the apparel industry in North Cyprus. David et al. (2006) only put emphasis on studying the relevance of APS principles in the particular area of the aluminium conversion industry. Hvolby and Kenn (2010) discussed the basic functionality of APS systems and stresses how essential it is for a good outcome that the user is familiar with the core APS functionality to enable a careful setup of the many (conflicting) planning parameters. Chen et al. (2011) presented a case study for the APS problem encountered in a light source manufacturer. Garcia-Sabater et al. (2012) presented an operations planning scheme based on mathematical programming models (mixed-integer linear programming models) integrated into a web-enabled APS, developed for and implemented in an engine assembler that supplies the car industry. Zhong et al. (2013) proposed an RFID-enabled real-time advanced production planning and scheduling shell (RAPShell) to coordinate different decision makers across production processes. Chen et al. (2017) developed an APS system for the 4.5th generation colour filter fabs, in which the tabu-search (TS) and SA algorithms were applied to optimise scheduling problems. Ivert and Jonsson (2010) explored what potential benefits may be achieved by using APS systems in the sales and operations planning process.

The APS system has received tremendous attentions in recent years (Tang et al., 2001; Lee et al., 2002; Metaxiotis et al., 2002; Chen et al., 2011; Kung and Chern, 2009; Ozturk and Ornek, 2016; Sobeyko and Mönch, 2016). However, very few results are available on real world cases. Unfortunately though, most of the previous research is based on assumptions that are different from the real manufacturing environment. However, the expected complexity and cost of and implementing APS system are still preventing the spread of such systems in more industries. Especially, the raw material of tobacco is processed in the stage of cut tobacco, which is the most important step in the tobacco industry. There are the most process, the longest process, the most complex 
operations and machines in this stage. The shop scheduling of cut tobacco is a complex nonlinear system, which determines the quality and efficiency of tobacco product. Although the planning cycle is short, the amount of information involved in the APS is large.

\section{Tobacco industrial problem}

Let us concentrate on a planning and scheduling problem in a company of the tobacco industry. The company produces different types of cigarettes according to the procedure whose description is shown in Figure 1. The cigarette paper and cut tobacco are wrapped and merged with tipping paper and filter tips to produce a cigarette. Filter paper and acetate are needed to produce the filter tips. The cut tobacco is generated from the raw tobacco.

Figure 1 Procedure of cigarette production (see online version for colours)

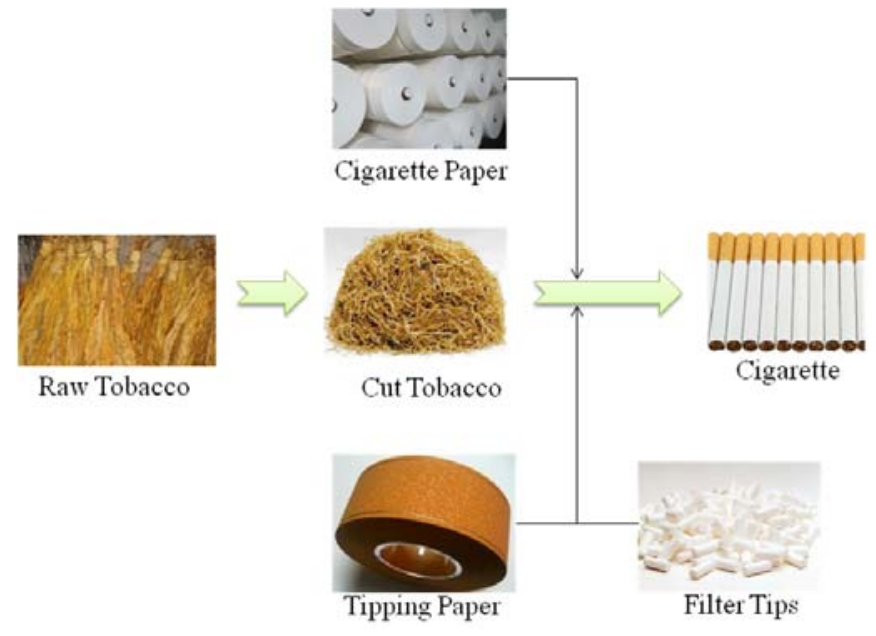

The above procedure can be divided into two main stages, the first stage is the cut tobacco production and the second stage is cigarette wrapping and packing. The cut tobacco is transferred to the cigarette wrapping and packing machines with parallel feeders. Therefore, the production quantity and delivery of cut tobacco is controlled by the requirements of cigarettes. And the permutation of producing batches must be arranged to satisfy the lead-time requirement in the second stage.

The cut tobacco production is an important stage of procedure, which includes the longest and most processes and requires the lots of equipment for the complicated processes. The cut tobacco production is a complex nonlinear system, which determines the quality and efficiency of cigarette production. There is an urgent demand for planning and scheduling optimisation of cut tobacco. Currently, the decisions that generate the planning and scheduling are carried out by human planners, which rely on the personal experience and ability. This lack of use of more APS might be related with the complexity of real constraints of cut tobacco in the tobacco industry. 
Table 1 Notation list

\begin{tabular}{|c|c|}
\hline Notation & Definition \\
\hline$i$ & Batch index \\
\hline$j$ & Shift index \\
\hline$l$ & Production line index \\
\hline$p \in[1, N P]$ & Index of process section (e.g., 1: cut leaf, 2: cut tobacco) \\
\hline$N L_{p}$ & Number of production lines in process section $p$. \\
\hline $\operatorname{MinST} T_{p, i}$ & Minimum storage time of batch $i$ in process section $p$. \\
\hline$N P$ & Number of process sections. \\
\hline$C N$ & Number of changeover in all production lines. \\
\hline$V_{l}$ & Speed of line $l$. \\
\hline$\underline{U_{p, l}}$ & Load of line $l$ in process section $p$. \\
\hline$S T \_S h i f t_{l, j}$ & Start time of $j$ shift in line $l$. \\
\hline ET_Shift $t_{l, j}$ & End time of $j$ shift in line $l$. \\
\hline$N B_{p}$ & Number of batch produced in process section $p$. \\
\hline$Q_{p, i}$ & Quantity of batch $i$ in process section $p$. \\
\hline$G_{p, i}$ & Brand grade of batch $i$ in process section $p$ \\
\hline$D T_{p, i}$ & Expected completion time of batch $i$ in process section $p$. \\
\hline$S T_{p, i}$ & Planned start time of batch $i$ in process section $p$ \\
\hline$E T_{p, i}$ & Planned completion time of batch $i$ in process section $p$. \\
\hline$x_{i, l}$ & If batch $\mathrm{i}$ was assigned to line $l, x_{i, l}$ otherwise $x_{i, l}=0$ \\
\hline pre $_{p, i}$ & Front batch index in process section $p$ corresponding batch $i$. \\
\hline
\end{tabular}

Take an example of the company we investigate, the process of cut tobacco is shown in Figure 2. As one can see, the process of cut tobacco includes opening, slicing, aging, bulking, casing, blending and so on. The type of constrains in the planning and scheduling of cut tobacco as follow:

- Process constraints. There are different attributes of process, such as KLK drying, HDT drying, SH93 drying and so on. The entire batch of cut tobacco can come out after coming in completely. For the formula processing mode, there is often one-to-one batch between leaf tobacco and cut tobacco, while there is a typical hybrid production for grouping processing mode.

- Time constraints. There is the minimum storage time for some bulkers. The planned completion time of each batch cannot be greater than the expected completion time of that. The planned time of each batch should be within the available shift time. First in and first out for bulkers, in order to minimise the storage time. Meanwhile, the planned time of batch must follow the factory calendar.

- Equipment constraints. There are some fixed connection between machines, such as feeders and cigarette wrapping machines, bulkers and feeders.

- Quantity constraints. The quantity of batch cannot be greater than the volume of bulker and be an integer multiple of certain unit. 
Figure 2 Process of cut tobacco

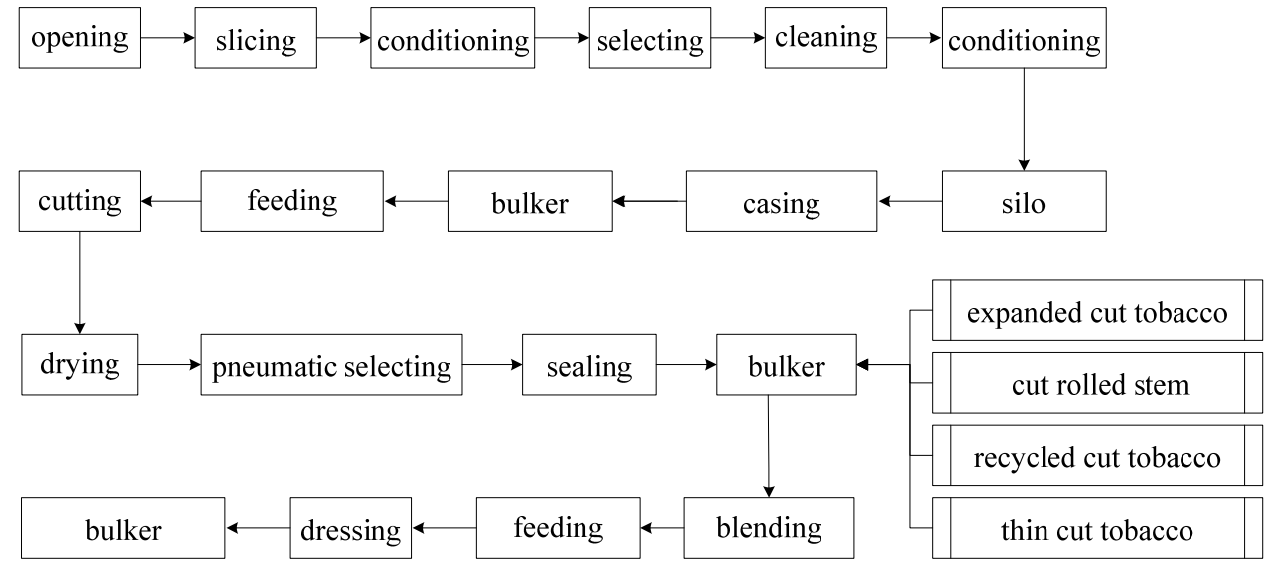

The planning and scheduling of cut tobacco is a typical combinatorial optimisation problem under above constraints. The APSoCT is to find an optimal machines and sequence of production so that the objectives are minimised or maximised. In this paper, the proposed APSoCT system is implied in the flow shop of cut tobacco.

To mathematically formulate the above-mentioned tobacco industrial problem, it is necessary to define the nomenclature presented in Table 1.

minimise $C N$

$\operatorname{minimise} \sum_{p=1}^{N P}\left(\max _{l} U_{p, l}-\min _{l} U_{p, l}\right)$

$\operatorname{minimise}\left(\max _{p \in[1, N P], i \in\left[1, N B_{p}\right]} E T_{p, i}-\min _{p \in[1, N P], i \in\left[1, N B_{p}\right]} S T p, i\right)$

Subject to:

$$
\begin{aligned}
& \forall i \in\left[1, N B_{p}\right], \forall p \in[2, N P], S T_{p, i}=\max \left(E T_{p-1, i}+\operatorname{MinST}_{p-1, i}, E T_{p, p r e p, i}\right) \\
& \forall i \in\left[1, N B_{p}\right], E T_{p, i}=S T_{p, i}+Q_{p, i} / \sum x_{i, l} v_{l} \\
& C N=\sum_{p=1}^{N P} \sum_{i=1}^{N B_{p}} 1\left\{G_{p, i}>G_{p, p_{p, i}} \& \& p r e_{p, i} \neq n u l l\right\} \\
& U p, l=\sum_{i \in\left[1, N B_{p}\right]} x_{i, l} Q_{p, i} \\
& \forall p \in[1, N P], i \in\left[1, N B_{p}\right], S T_{p, i} \geq \sum_{l \in\left[1, N L_{p}\right]} x_{i, l} S T_{-} S_{\text {Shift }} t_{l, j}
\end{aligned}
$$




$$
\begin{aligned}
& \forall p \in[1, N P], i \in\left[1, N B_{p}\right], E T_{p, i} \geq \sum_{l \in\left[1, N L_{p}\right]} x_{i, l} E T_{-} S_{\text {Shift }} t_{l, j} \\
& \forall i \in\left[1, N B_{N P}\right], E T_{N P, i}+\operatorname{MinST}_{N P, i} \leq D T_{N P, i}
\end{aligned}
$$

In the above mathematical model, the expression (1) is to minimise the number of changeover in all production lines; the expression (2) is to minimise the load difference between lines in the process section; the expression (3) is to minimise the completion time of batch of cut tobacco; the expression (4) ensures the batch $i$ in process section $p$ would start after the minimum storage time and the front batch; the expression (5) calculates the planned completion time of batch $i$ in process section $p$; the expression (6) calculates the number of changeover at the same shift; the expression (7) calculates the load of line $l$ in process section $p$; the expressions (8) and (9) denote that the planned time of each batch should be within the available shift time; the expression (10) guarantees that the available batches in the last process section NP must be finished before the expected completion time corresponding to batches.

\section{Design of APSoCT}

\subsection{Function modules of APSoCT}

The function modules of APSoCT can be divided into three sections, as shown in Figure 3:

- Basic function module. User right management, factory and equipment management, material management, BOM management, process management and so on. The module can provide the basic data and system right setting for APSoCT.

- Planning function module. Planning rules management (optimisation algorithm setting, optimisation objectives setting, constraint rules setting), wrapping planning management, cut tobacco batch management, planning and scheduling of cut tobacco and so on. The module is the core of APSoCT.

- View function module. Gantt chart management, data report management, process route planning management and multi-objective optimisation management and so on. The module can provide intuitive and friendly view display to planners.

\subsection{Scheduling process of APSoCT}

APSoCT can optimise the planning and scheduling of cut tobacco batches according to the cigarette production planning, as shown in Figure 4. APS supports decision making to reduce costs and inventory and increase manufacturing throughput and improve productivity. For this support, APSoCT uses optimisation techniques to model and determine the quantities to be produced, stored, transported and procured by respecting real constraints. 
Figure 3 Function modules of APSoCT (see online version for colours)

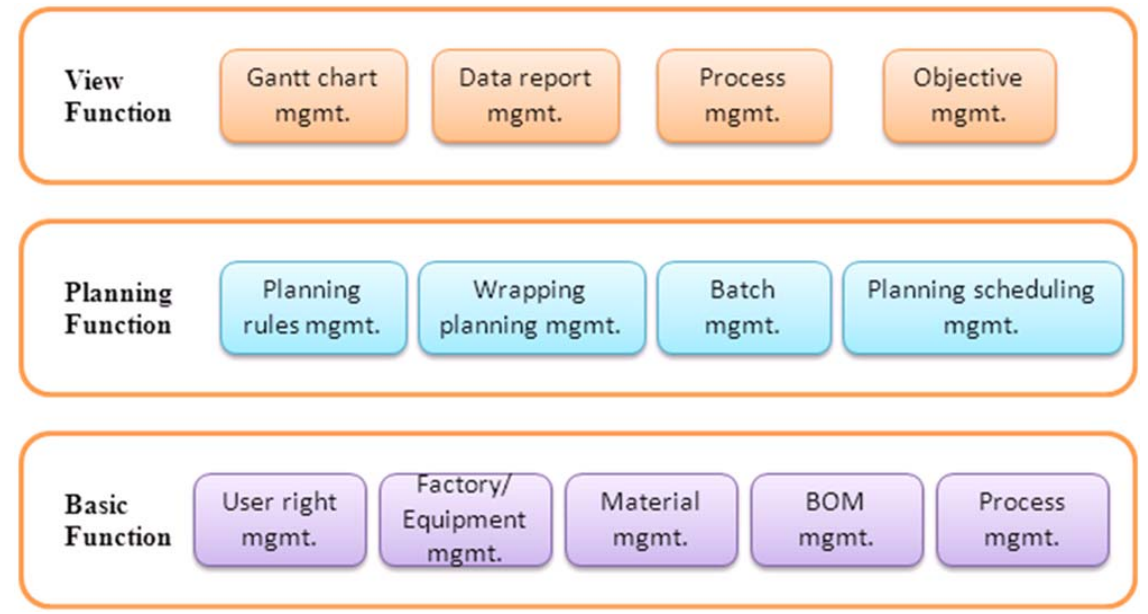

Figure 4 Scheduling process of APSoCT

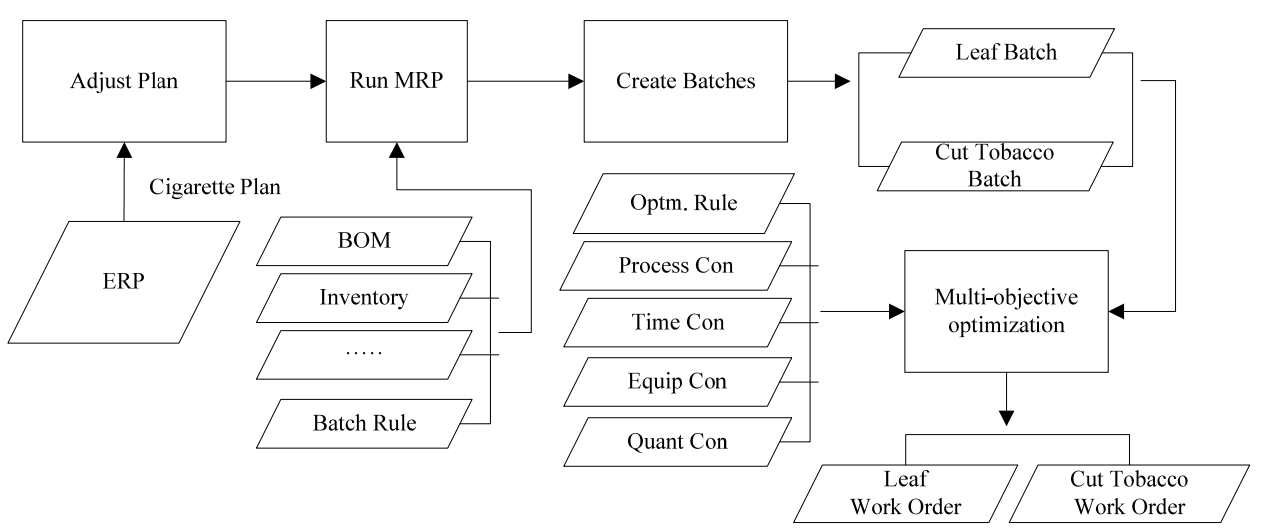

APSoCT would extract data of cigarette production planning from ERP system, which can be modified by planners. The data of cigarette production planning is the basis of the creating and optimising of batches of cut tobacco. Then, APSoCT runs MRP, according to the BOM of cigarette products and material stock, batch rules and so on. After MRP running, the batches of cut tobacco are created in real-time. Next step is optimising these batches. By respecting real process, time, equipment, quantity constraints, a multi-objective optimisation can be made and explored by APSoCT. 


\section{Design of heuristics}

\subsection{GA for APSoCT}

During the last decade, GA has been widely applied to many engineering fields, especially in the production scheduling field (Wang and Zheng, 2003). GA-exhibit parallelisms contain certain redundant and historical information of the past solutions and are suited to implementation for large parallel architectures (Goldberg, 1989). GAs are search algorithms based on the mechanics of natural selection and natural genetics. They work with a population of solutions and attempt to guide the search toward improvement, using a survival of the fittest principle.

In general, GA consists of the following steps, as shown in Figure 5:

Step 1 Initialise a population of $N_{p o p}$ chromosomes where $N_{p o p}$ is the number of chromosomes in each population.

Step 2 Evaluate the values of the objective functions of each chromosome.

Step 3 Calculate the fitness value of each chromosome by using the weighted sum approach. Select a pair of chromosomes from the current population according to the selection probability. This step is repeated times to produce $N_{p o p}$ offspring by the crossover operation in Step 4.

Step 4 For each selected pair, apply a crossover operation to generate an offspring with the crossover probability. $N_{p o p}$ chromosomes should be generated by the crossover operation.

Step 5 For each chromosome generated by the crossover operation, apply a mutation operation with a prespecified mutation probability.

Step 6 Adopt elitist strategy. Insert the best chromosome into the current population by removing the worst chromosome (having max. fitness value).

Step 7 If the termination condition is satisfied, stop and return the best chromosome; otherwise, go to Step 2.

\subsection{Individual representation}

In this paper, a chromosome represents a feasible solution in the solution space. Based on the lines and the batches of cut tobacco, an encoding approach for the APSoCT is presented. Suppose that the cut tobacco is required to pass $N P$ process stages and $N B_{p}$ batches are to be processed for some stage $p \in[1,2, \ldots, P]$. The length of chromosome is $2 \times \sum_{p=1}^{N P} N B_{p}$, which is divided into two parts: Line permutation and Priority permutation. The first $\sum_{p=1}^{N P} N B_{p}$ genes indicate the selected line identifier for the corresponding batch in the integer numbers, while the later $\sum_{p=1}^{N P} N B_{p}$ genes indicate the priority of the corresponding batch in the real numbers. 
Figure 5 Outline of GA for APSoCT (see online version for colours)

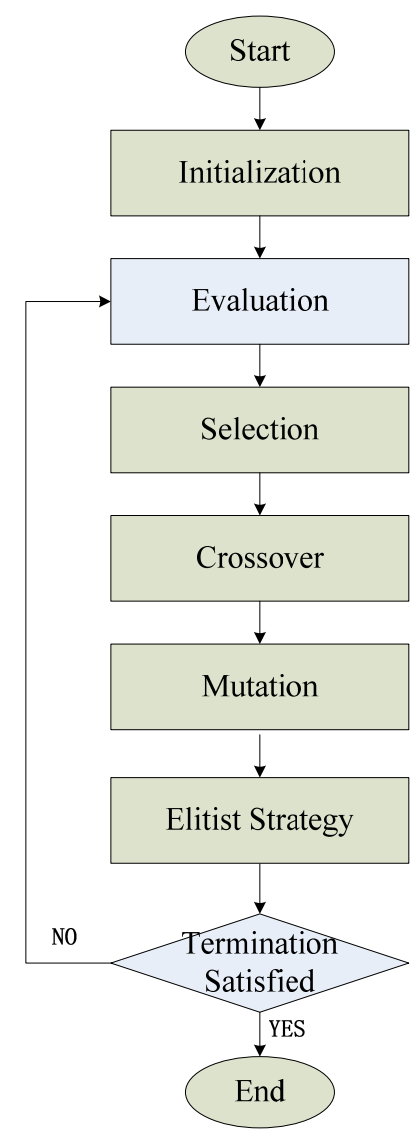

\subsection{Individual decoding}

When we evaluate a chromosome, the decoding procedure needs to be employed. The decoding procedure includes two steps: the first step is to decode the line permutation of chromosome (shown in Figure 6) and then the second is to decode the priority permutation (shown in Figure 7).

\subsection{Example}

Assume that eight batches are scheduled at three process stages in a cut tobacco shop. Table 2 is the line identifiers for each batch. Three batches are included in the 1st process stage ( $p=1, i=1,2,3)$, three batches are included in the 2 nd process stage ( $p=2, i=4$, $5,6)$ and two batches are included in the 3rd process stage $(p=3, i=7,8)$. In Figure 6 , there is an example of chromosome $\pi=[(2,1,1,2,1,2,1,1),(067,0.34,0.14,0.45$, $0.32,0.01,0.23,0.78)]$. The line identifier of the 1 st batch is 2 and the priority of the 1 st batch is 0.67 . 
Figure 6 Procedure 1 for decoding of line permutation

Procedure 1: decoding (line permutation)

Input: matrix of candidate lines $T$, chromosome $C h r$, number of batches $\sum_{p=1}^{N P} N B_{p}$.

Output: feasible line sequence $F L S$.

Begin

$F L S \leftarrow \Phi$

for $\left(i=0 ; i<\sum_{p=1}^{N P} N B_{p} ; i++\right)$

the $i^{\text {th }}$ gene of chromosome $C h r[i]$;

for $\left(l=0 ; l<\sum_{p=1}^{N P} N l_{p} ; l++\right)$

if $(T[i, l]=C h r[i])$

\{

$F L S \leftarrow F L S \cup l$

break;

\}

\}

End

Output feasible line sequence $F L S$.

Take an example of Figure 8 and Table 2, the line identifier of batch $i=1$ is 2 and the candidate lines for batch $i=1$ have two ones L1 and L2 with the identifiers 1 and 2. As shown in Figure 9, the selected line for batch $i=1$ is L2. Likewise, the selected one for batch $i=2$ is L2 and the selected one for batch $i=3$ is L1. Note that although the line identifiers of two batches are the same, the corresponding lines could be different. In order to illustrate the decoding procedure, the genes with the same lines are in the same background colour. If there are the genes with the same background colour, the batches need to be sequenced according to the corresponding priority. The higher priority is, the batch would come into production early. For instance of batches $i=4$ and $i=6$, these batches are produced in the same line L4. The batch $i=4$ will be produced earlier than batch $i=6$, because the priority of batch $i=4$ is 0.45 , which is larger than that of batch $i=6(0.01)$. 
Figure 7 Procedure 2 for decoding of priority permutation

Procedure 2:decoding (priority permutation)

Input: feasible line sequence $F L S$, chromosome $C h r$, number of batches $\sum_{p=1}^{N P} N B_{p}$.

Output: feasible batch sequence $F B S$

Begin

$F B S \leftarrow \Phi$

Multi-dimensional array $M \leftarrow \Phi$;

for $\left(i=0 ; i<\sum_{p=1}^{N P} N B_{p} ; i++\right)$

\{

$M[i, 0]=i$;

$M[i, 1]=F L S[i]$

$M[i, 2]=C h r\left[i+\sum_{p=1}^{N P} N B_{p}\right]$;

$M[\underline{i}, 3] \leftarrow \phi ;$

\}

sort array $M$ by the values of the second column and the third column of array $M$;

set the line code $l c=M[0,1]$;

set the batch sequence index $b s=1$;

for $\left(i=0 ; i<\sum_{p=1}^{N P} N B_{p} ; i++\right)$

\{

set the line code $l c^{\prime}=M[i, 1]$;

if $\left(l c==l c^{\prime}\right)$

\{

$M[i, 3]=b s$

update $b s \leftarrow b s+1$;

\}

else

\{

update $b s \leftarrow 1$;

$M[i, 3]=b s$;

\}

$F B S \leftarrow F B S \cup M[i, 3] ;$

\}

End

Output feasible batch sequence $F B S$. 
Figure 8 Structure of proposed chromosome

\begin{tabular}{|c|c|c|c|c|c|c|c|c|}
\hline \multirow{2}{*}{$\begin{array}{l}\text { Process Section } \\
\text { Batch Index }(i)\end{array}$} & \multicolumn{3}{|c|}{$\overbrace{}^{p=1}$} & \multicolumn{3}{|c|}{$\underbrace{p=2}$} & \multicolumn{2}{|c|}{$\underbrace{p=3}$} \\
\hline & 1 & 2 & 3 & 4 & 5 & 6 & 7 & 8 \\
\hline \multirow{2}{*}{$\begin{array}{l}\text { Chromosome } \\
\text { (16 genes) }\end{array}$} & 2 & 1 & 1 & 2 & 1 & 2 & 1 & 1 \\
\hline & 0.67 & 0.34 & 0.14 & 0.45 & 0.32 & 0.01 & 0.23 & 0.78 \\
\hline
\end{tabular}

Figure 9 Example of crossover scheme (see online version for colours)

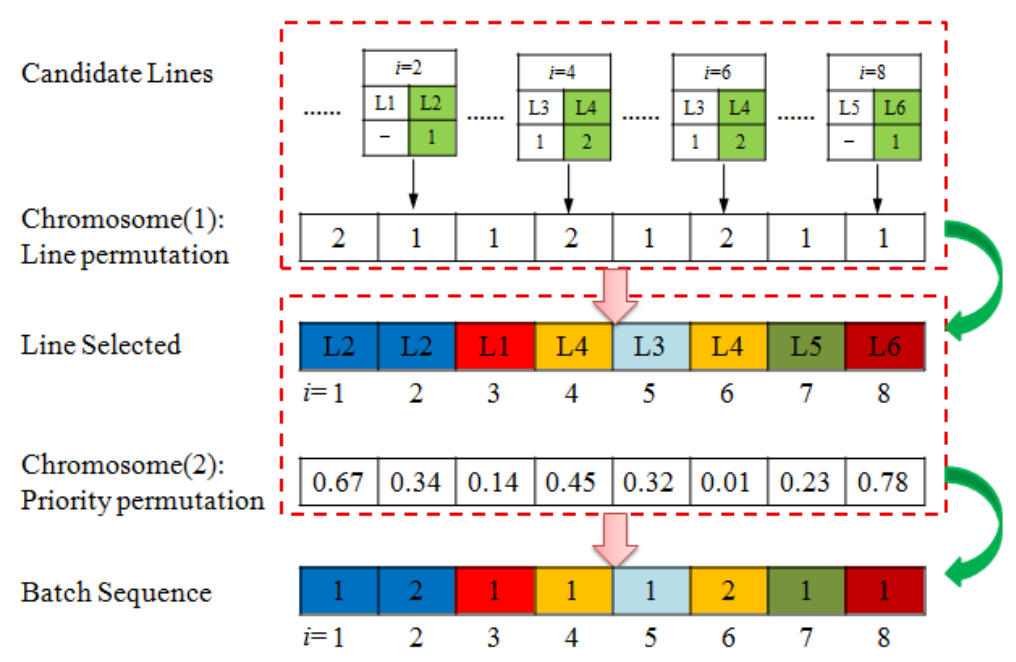

Table 2 Table of candidate lines

\begin{tabular}{lccccccc}
\hline \multirow{2}{*}{$\begin{array}{l}\text { Process } \\
\operatorname{section}(p)\end{array}$} & $\begin{array}{c}\text { Batch } \\
\text { index }(i)\end{array}$ & L1 & L2 & L3 & L4 & L5 & L6 \\
\hline$p=1$ & 1 & 1 & 2 & - & - & - & - \\
& 2 & - & 1 & - & - & - & - \\
& 3 & 1 & - & - & - & - & - \\
& 4 & - & - & 1 & 2 & - & - \\
$p=1$ & 5 & - & - & 1 & 2 & - & - \\
& 6 & - & - & 1 & 2 & - & - \\
$p=1$ & 7 & - & - & - & - & 1 & 2 \\
& 8 & - & - & - & - & - & 1 \\
\hline
\end{tabular}

Note: '-' denotes the corresponding line unavailable for the batch.

\section{Development of APSoCT}

Both browser/server and client/server architecture is adopted for the APSoCT architecture, which combines the advantages of both. The architecture includes three layers: user layer, business logic layer and data layer, as shown in Figure 10. The optimisation heuristic runs on high performance server. The user layer provides the 
succinct human-machine interactive interface, which responds to the users' requests. The business logic layer completes the computation and logical processing. And the date layer provides the storage service. Coupling is dramatically reduced between the business logic layer and the data layer.

Figure 10 Architecture of APSoCT (see online version for colours)

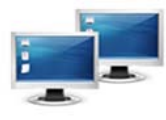

User layer

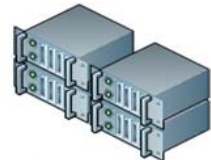

Business logic layer

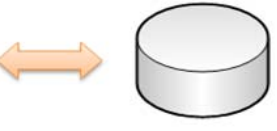

Data layer

Figure 11 Software interface of APSoCT (see online version for colours)

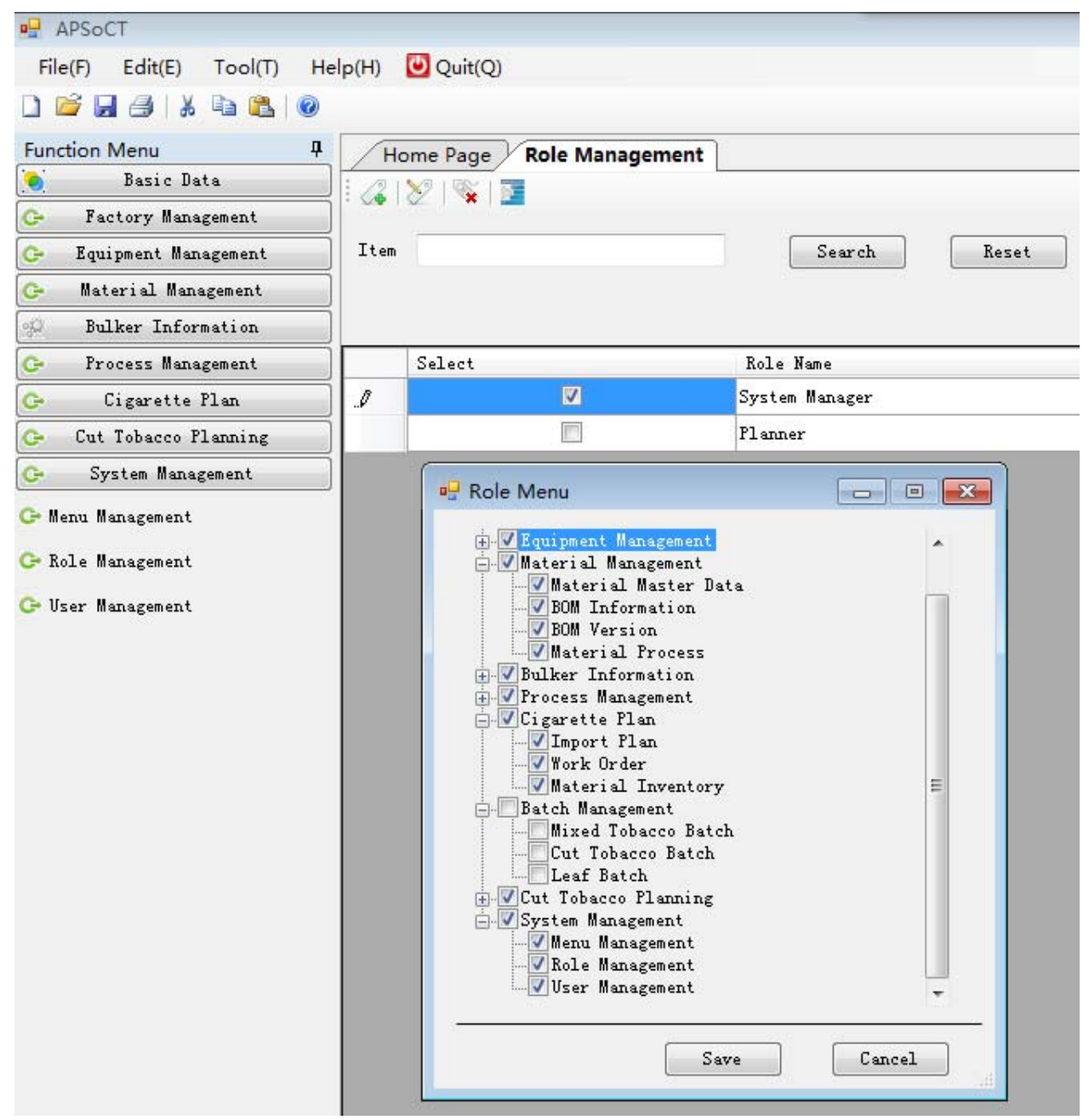


The APSoCT system employs the object-oriented high-level language $\mathrm{C \#}$ and the database SQL server. C\# is a simple, modern, object-oriented and type-safe programming language. It will immediately be familiar to $\mathrm{C}$ and $\mathrm{C}++$ programmers. $\mathrm{C} \#$ combines the high productivity of rapid application development (RAD) languages and the raw power of $\mathrm{C}++$. It is developed by Microsoft within its .NET initiative. SQL server is a relational database management system developed by Microsoft.

Figure 12 Cut tobacco planning of APSoCT (see online version for colours)

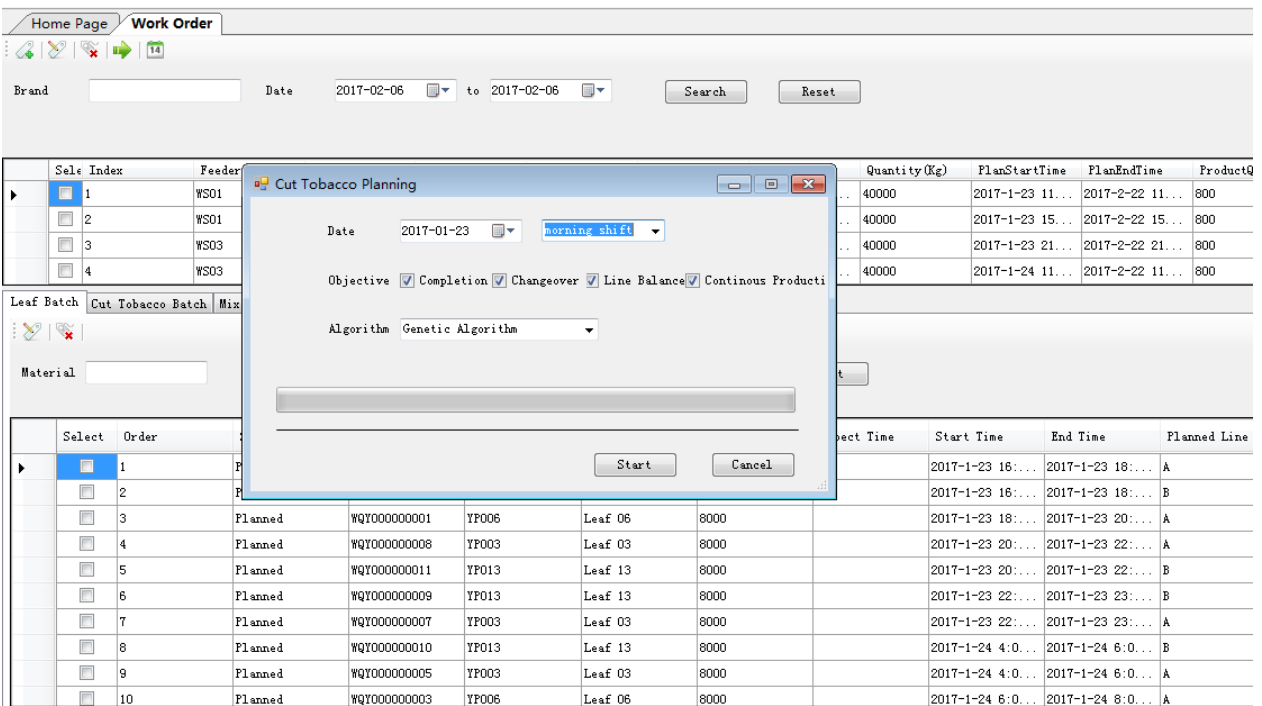

Figure 13 Gantt chart of APSoCT (see online version for colours)

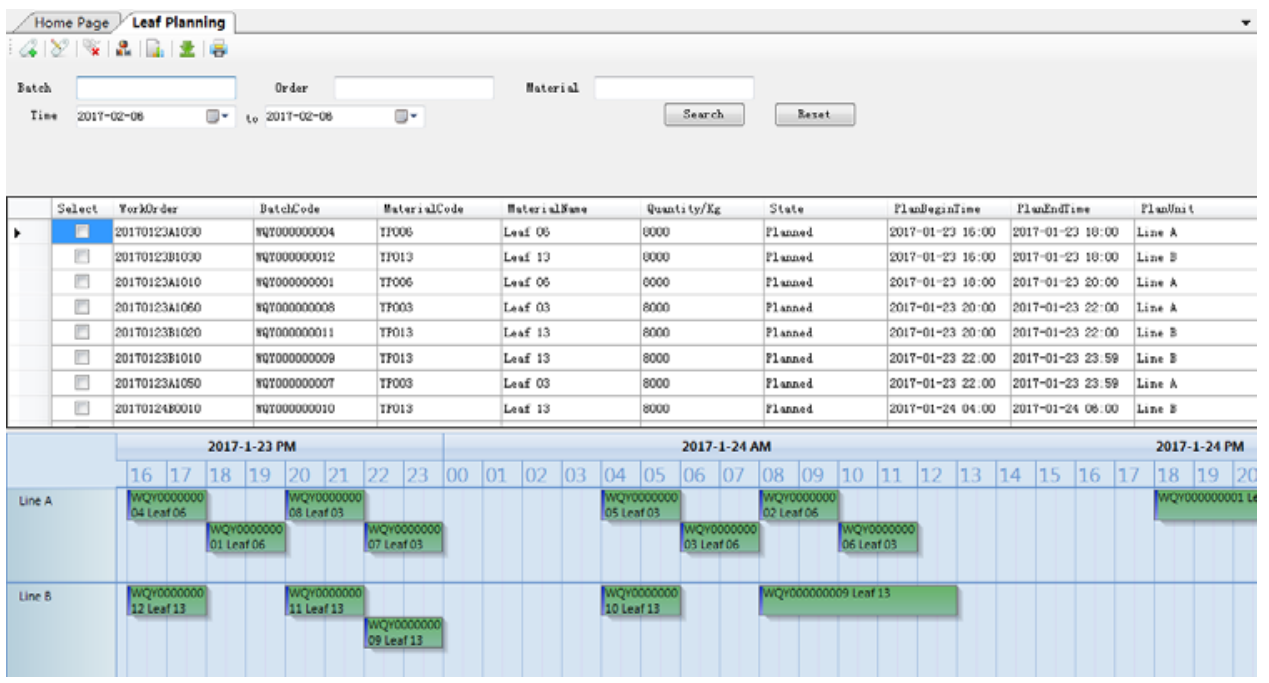


Figure 11 shows the software interface of APSoCT, especially the function menu including the basic data, factory management, equipment management, material management, bulker information, process management, cigarette plan, cut tobacco planning, system management.

Figure 12 introduces the cut tobacco planning interface of APSoCT, where planners can set some optimisation parameters, such as the planned date and shift of production, the optimised objectives and the used heuristic.

In Figure 13, a Gantt chart is drawn to clearly visualise the planning result of cut tobacco by APSoCT, meanwhile the report of optimisation result is shown.

Figure 14 provides a graphical representation of the optimised objective (the utilisation of facilities), while Figure 15 provides a graphical representation of the optimised objective (the equilibrium of shifts).

Figure 14 Graph of the facilities utilisation (see online version for colours)

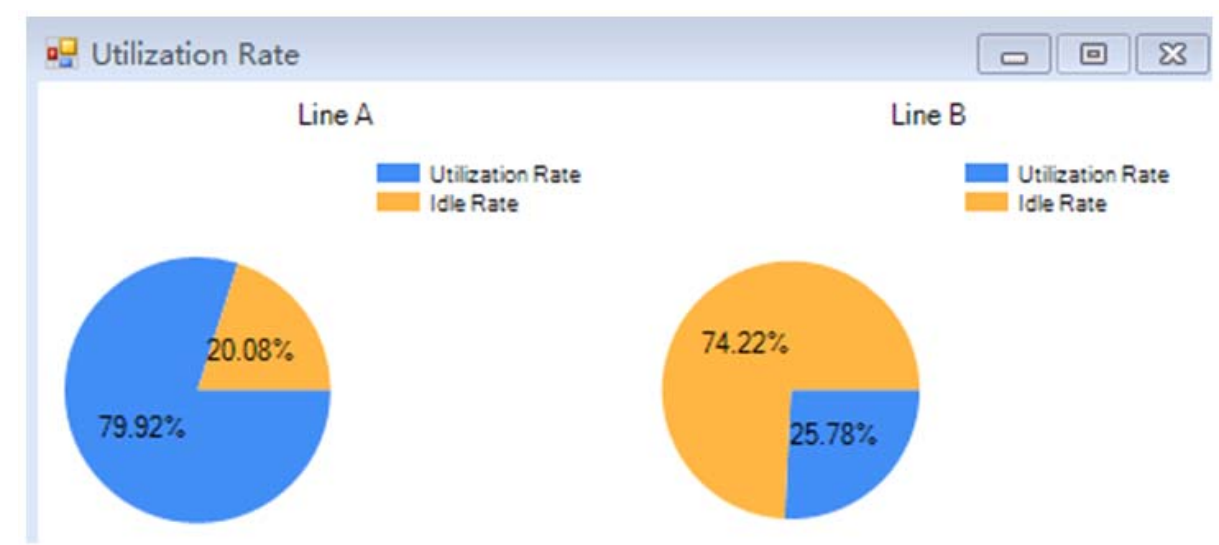

Figure 15 Graph of the shift equilibrium (see online version for colours)

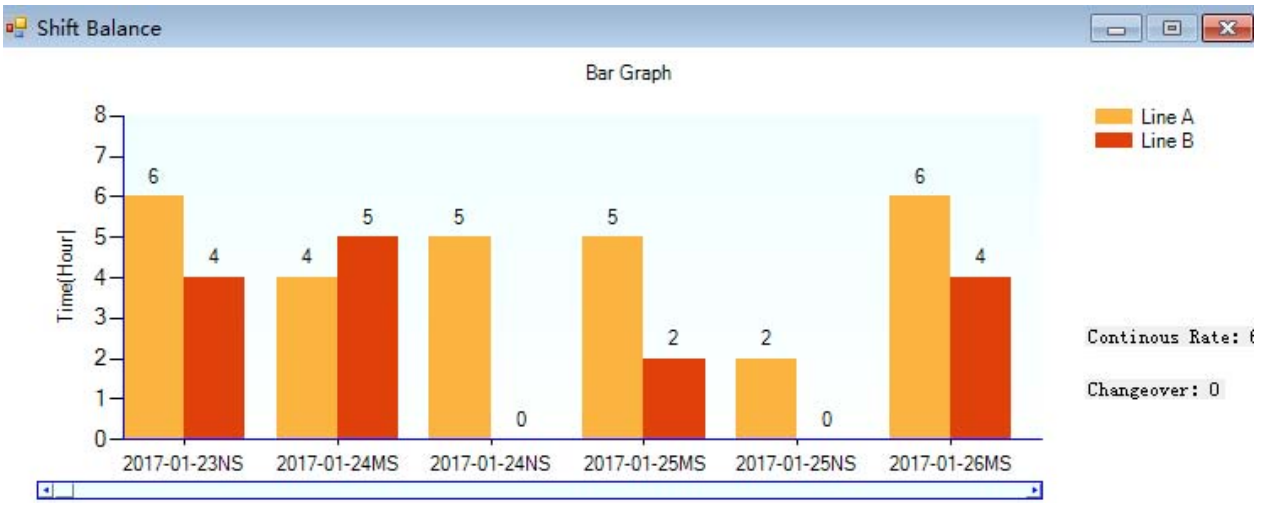




\section{Conclusions}

This paper presented has described the APS system for the tobacco industry. The reasonable production plan would contribute to making use of the limited resources, raising the production efficiency, reducing the production cost and saving energy. Software called APSoCT has been developed for the planning and scheduling of cut tobacco using an object-oriented paradigm. Benefits of implementing the APSoCT system are as follows:

- Prior to accepting an order, the APSoCT provides management with the ability to estimate due dates. Thus, it is possible to make suggestions to modify orders so that it will be favourable to both parties.

- The reasonable planning and scheduling of each process section could considerably shorten the storage-time and result in lower costs.

- The company ensures that the accessories are received on time by arranging delivery schedules that correspond to the cut tobacco processes.

- Fewer manual mistakes are committed when using the APSoCT and engineers can save their working time.

It is worthwhile pointing out some difficulties and shortcomings which arose in the current version of APSoCT:

- Difficulties in obtaining a specific priority level of multi-objectives.

- Inaccuracy of product data. The APSoCT will be integrated with other systems, such as ERP and MES.

A future research line would be to develop multiple algorithms that solve the problem to estimate performance, such as particle swarm optimisation, ant colony optimisation and differential evolution algorithms, etc. Together, as a future research activity, self-adaption parameters of algorithms would be studied without the setting for planners.

\section{Acknowledgements}

The authors are very much thankful to all participants in Beihang University. This paper is partially supported by National Natural Science Fund of China (No. 51705472); Aviation Foundation of China (No. 2015ZG55018); Foundation for Key Research in Higher Education of Henan Province (No. 18A460033, 18A460034). The authors are also very much thankful to all reviewers and referees for their constructive criticisms and suggestions that helped to improve this paper.

\section{References}

Caridi, M., Sianesi, A., Milano, P.D. and Vinci, P.L.D. (2000) 'Trends in planning and control systems: APS-ERP integration', International Conference on Global Production Management, Vol. 24, pp.105-111.

Chen, I.J. (2001) 'Planning for ERP systems: analysis and future trend', Bus. Process Management Journal, Vol. 7, No. 5, pp.374-386. 
Chen, J.C., Chen, Y.Y., Chen, T.L. and Lin, J.Z. (2017) 'Comparison of simulated annealing and tabu search algorithms in advanced planning and scheduling systems for TFT-LCD colour filter fabs', International Journal of Computer Integrated Manufacturing, Vol. 30, No. 6, pp.1-19.

Chen, K.J., Ji, P. and Wang, Q. (2011) 'A case study for advanced planning and scheduling (APS)', Journal of Systems Science and Systems Engineering, Vol. 20, No. 4, pp.460-474.

Cheng, B.Y., Yang, S.L., Hu, X.X. and Li, K. (2014) 'Scheduling algorithm for flow shop with two batch-processing machines and arbitrary job sizes', International Journal of Systems Science, Vol. 45, No. 3, pp.571-578.

Dai, M., Tang, D.B., Giret, A., Salido, M.A. and Li, W.D. (2013) 'Energy-efficient scheduling for a flexible flow shop using an improved genetic-simulated annealing algorithm', Robotics and Computer-Integrated Manufacturing, Vol. 29, No. 5, pp.418-429.

David, F., Pierreval, H. and Caux, C. (2006) 'Advanced planning and scheduling systems in aluminum conversion industry', International Journal of Computer Integrated Manufacturing, Vol. 19, No. 7, pp.705-715.

Garcia-Sabater, J.P., Maheut, J. and Garcia-Sabater, J.J. (2012) 'A two-stage sequential planning scheme for integrated operations planning and scheduling system using MILP: the case of an engine assembler', Flexible Services and Manufacturing Journal, Vol. 24, No. 2, pp.171-209.

Goldberg, D.E. (1989) Genetic Algorithms in Search, Optimization and Machine Learning, Addison Wesley Longman Publishing Co., New Jersey.

Hashemipour, M. (2004) 'Integration of process planning and scheduling for outsourcing in the apparel industry', The Journal of the Textile Institute, Vol. 95, No. 1, pp.9-18.

Hvolby, H.H. and Kenn, S.J. (2010) 'Technical and industrial issues of advanced planning and scheduling (APS) systems', Computers in Industry, Vol. 61, No. 9, pp.845-851.

Ivert, L.K. and Jonsson, P. (2010) 'The potential benefits of advanced planning and scheduling systems in sales and operations planning', Industrial Management and Data Systems, Vol. 110, No. 5, pp.659-681.

Kung, L.C. and Chern, C.C. (2009) 'Heuristic factory planning algorithm for advanced planning and scheduling', Computers and Operations Research, Vol. 36, No. 9, pp.2513-2530.

Lee, Y.H., Jeong, C.S. and Moon, C. (2002) 'Advanced planning and scheduling with outsourcing in manufacturing supply chain', Computers and Industrial Engineering, Vol. 43, Nos. 1-2, pp.351-374.

Lei, D.M. and Wang, T. (2011) 'An effective neighborhood search algorithm for scheduling a flow shop of batch processing machines', Computers and Industrial Engineering, Vol. 61, No. 3, pp.739-743.

Lin, C.H., Hwang, S.L. and Wang, E.M.Y. (2007) 'A reappraisal on advanced planning and scheduling systems', Industrial Management and Data Systems, Vol. 107, No. 8, pp.1212-1226.

Liu, D.Y., Pu, Y. and Ji, Y. (2009) 'Development of a multi-objective GA for advanced planning and scheduling problem', International Journal of Advanced Manufacturing Technology, Vol. 42, Nos. 9-10, pp.974-992.

Metaxiotis, K.S., Askounis, D. and Psarras, J. (2002) 'Expert systems in production planning and scheduling: a state-of-the-art survey', Journal of Intelligent Manufacturing, Vol. 13, No. 4, pp.253-260.

Moon, C., Kim, J.S. and Gen, M. (2004) 'Advanced planning and scheduling based on precedence and resource constraints for e-plant chains', International Journal of Production Research, Vol. 42, No. 15, pp.2941-2954.

Ozturk, C. and Ornek, M.A. (2016) 'Optimisation and constraint based heuristic methods for advanced planning and scheduling systems', International Journal of Industrial Engineering, Vol. 23, No. 1, pp.26-48. 
Pongcharoen, P., Hicks, C. and Braiden, P.M. (2004) 'The development of genetic algorithms for the finite capacity scheduling of complex products with multiple levels of product structure', European Journal of Operational Research, Vol. 152, No. 1, pp.215-225.

Sobeyko, O., and Mönch, L. (2016) 'Integrated process planning and scheduling for large-scale flexible job shops using metaheuristics', International Journal of Production Research, Vol. 55, No. 2, pp.392-409.

Tang, L.X., Liu, J.Y., Rong, A.Y. and Yang, Z.H. (2001) 'A review of planning and scheduling systems and methods for integrated steel production', European Journal of Operational Research, Vol. 133, No. 1, pp.1-20.

Wang, L. and Zheng, D.Z. (2003) 'An effective hybrid heuristic for flow shop scheduling', International Journal Advanced Manufacturing Technology, Vol. 21, No. 1, pp.38-44.

Yun, Y.S. and Gen, M. (2002) 'Advanced scheduling problem using constraint programming techniques in SCM environment', Computers and Industrial Engineering, Vol. 43, Nos. 1-2, pp.213-229.

Zhong, R.Y., Li, Z., Pang, L.Y., Pan, Y., Qu, T. and Huang, G.Q. (2013) 'RFID-enabled real-time advanced planning and scheduling shell for production decision making', International Journal of Computer Integrated Manufacturing, Vol. 26, No. 7, pp.649-662. 